https://doi.org/10.15407/ujpe64.11.1078

O.S. STASHKO, V.I. ZHDANOV

Taras Shevchenko National University of Kyiv

(60, Volodymyrs'ka Str., Kyiv 01033 Ukraine; e-mail: alexander.stashko@gmail.com;

valeryzhdanov@gmail.com)

\title{
BLACK HOLE MIMICKERS IN ASTROPHYSICAL CONFIGURATIONS WITH SCALAR FIELDS ${ }^{1}$
}

\begin{abstract}
We study static spherically symmetric configurations of General Relativity in the presence of one scalar field (SF). For a monomial SF potential, the solutions of the Einstein SF equations are obtained numerically; then we get distributions of stable circular orbits around the configuration and build images of the corresponding "accretion disks" from the perspective of a distant observer. We discuss also a similar problem in the case of analytic solution with a specially selected SF potential that allows the existence of a black hole. We show that the images are similar in many cases to the case of ordinary Schwarzschild black hole with a luminous ring and a dark spot at the center. On the other hand, a certain selection of model parameters leads to specific features in these images that may help to exclude some exotic models.

Ke ywords: relativistic astrophysical objects, scalar fields, accretion disks.
\end{abstract}

\section{Introduction}

Recent publications of the Event Horizon Telescope (EHT) results [1] have increased interest in considerations of the black hole (BH) mimickers that can produce similar images from the perspective of a distant observer. In fact, within the EHT angular resolution, it is difficult to rule out a considerable number of alternative astrophysical objects (see, e.g., $[2,3]$ and references therein). Models of such mimickers may appear in alternative gravity theories with additional cosmological fields and within approaches to the dark energy problem [4-8]. The main question is whether it is possible to distinguish between different images such as that of a black hole and a naked singularity? In this paper, we discuss this issue in the framework of models that involve one scalar field (SF).

It is important to note that any introduction of arbitrarily small SF can strongly affect the spacetime topology. Indeed, under certain general conditions, a stationary black hole cannot be endowed with the scalar "hair" $[9,10]$ yielding the naked singularity. This is directly visible in the case of the Fisher solution (see [11]; see also [12-14]). However, despite these features, the observational manifestations of the

(c) O.S. STASHKO, V.I. ZHDANOV, 2019

1078 compact object with SF may be the same as in the case of a normal Kerr BH. This needs the investigation of a space-time geodesic structure around these objects with focus on the stable circular orbits (SCO) and the observed accretion disk images.

The circular orbits around relativistic astrophysical objects were studied in different contexts; an incomplete list includes, e.g., [16-23] and references therein. In this work, we consider a special problem dealing with a static spherically symmetric asymptotically flat configuration of General Relativity in the presence of one real SF, which is minimally coupled to gravity. Our SF model uses the Lagrangian with the canonical kinetic term.

In Section 2, we describe the basic relations and notations. In Section 3, we deal with numerical solutions for a monomial SF potential; then we study the SCO distributions (Section 4) and the related images corresponding to these distributions (Section 5 ). The monomial potential excludes the occurrence of the $\mathrm{BH}$ event horizons $[9,10]$. So, for a comparison in Section 6, we consider an alternative model from our paper [18], which admits this case.

1 This work is based on the results presented at the XI BolyaiGauss-Lobachevskii (BGL-2019) Conference: Non-Euclidean, Noncommutative Geometry and Quantum Physics.

ISSN 2071-0194. Ukr. J. Phys. 2019. Vol. 64, No. 11 


\section{Basic Relations}

We consider one minimally coupled real SF $\phi$ described by the Lagrangian density

$L=\frac{1}{2} \phi, \mu \phi^{, \mu}-V(\phi)$.

In this paper, we consider two types of SF potentials. In Sections 3, 4, and 5, which make up the bulk of the work, we deal with the monomial potential

$V(\phi)=w \phi^{2 n}, \quad w>0, \quad n=2,3, \ldots$

(massless case); the case of a linear massive scalar field $(n=1)$ has been studied in [18]. In Section 6, we consider an analytic model that admits the $\mathrm{BH}$ configurations.

The space-time endowed with the metric $g_{\mu \nu}$ subject to the Einstein equations is assumed to be asymptotically flat. In Sections 3, 4, and 5, we consider the static spherically symmetric space-time metric in curvature coordinates

$d s^{2}=e^{\alpha(r)} d t^{2}-e^{\beta(r)} d r^{2}-r^{2} d O^{2}$,

where $d O^{2}=d \theta^{2}+(\sin \theta)^{2} d \varphi^{2}$ is the interval on a unit sphere. The Einstein SF equations yield the closed system:

$\frac{d}{d r}\left[r\left(e^{-\beta}-1\right)\right]=-8 \pi r^{2} T_{0}^{0}$,

where $T_{0}^{0}=e^{-\beta} \phi^{\prime 2} / 2+V(\phi)$,

$r e^{-\beta} \frac{d \alpha}{d r}+e^{-\beta}-1=-8 \pi r^{2} T_{1}^{1}$,

$T_{1}^{1}=-e^{-\beta} \phi^{2} / 2+V(\phi)$, and

$\frac{d}{d r}\left[r^{2} e^{\frac{\alpha-\beta}{2}} \frac{d \phi}{d r}\right]=r^{2} e^{\frac{\alpha+\beta}{2}} V^{\prime}(\phi)$.

In view of the asymptotic flatness, we assume

$\lim _{r \rightarrow \infty}\left[r\left(e^{\alpha}-1\right)\right]=\lim _{r \rightarrow \infty}\left[r\left(e^{-\beta}-1\right)\right]=-r_{g}$,

where $r_{g}=2 M$, and $M>0$ is the configuration mass. We assume that $\phi(r) \rightarrow 0$ for $r \rightarrow \infty$ and

$\left|r^{2} \frac{d \phi}{d r}\right|<K_{1}<\infty$

\section{Numerical Solutions}

In order to find the solutions numerically, we start at sufficiently large values of the radial variable and then perform the integration backward for smaller values of $r$. For this, we need the initial data that have been obtained from asymptotic solutions for $r \rightarrow \infty$ corresponding to small field values $(\phi \rightarrow 0)$. One can show that $\phi(r)$ and $\phi^{\prime}(r)$ do not change their signs on $(0, \infty)$. So, we shall further assume that $\phi(r)>0$ and this function is monotonically decreasing.

As $r \rightarrow \infty$, the SF asymptotic expansions must be similar to those in the case of a flat space. The leading terms of the asymptotic expansion for $\phi$ can be looked for in a power-law form (except the case $n=2$, when logarithms are involved). In the zeroth approximation, we can write $\phi(r) \approx Q / r, \exp (\alpha)=\left(1-r_{g} / r\right)$, and $\exp (\beta)=\left(1-r_{g} / r\right)^{-1} \approx 1+r_{g} / r$. For $n=2$, the asymptotics is $\phi(r)=Q /(r \sqrt{\ln r})$. These formulas are sufficient to get the initial conditions for the numerical integration, though we checked our calculations by considering higher-order terms of expansion in powers of $1 / r$.

The choice of units allows us to fix $w=1$. Using the asymptotic relations, the solutions are continued to all values of $r>0$, and the spherically symmetric static asymptotically flat configuration is uniquely defined by constants $M, Q$. The qualitative behavior of the solutions for different parameters is essentially the same as that in the case of a linear massive scalar field $[15,18]$. For $r \rightarrow 0$, we have that $e^{\alpha} \gg e^{\beta}$ tends to zero and $\phi \rightarrow \infty$. This means that the singularity is physical, as it cannot be eliminated by a coordinate transformation.

\section{Test Particle Motion}

The test particle motion in the spherically symmetric space-time is considered in a standard way using the integrals for trajectories in the plane $\theta=\pi / 2$ :

$e^{\alpha}\left(\frac{d t}{d \tau}\right)^{2}-e^{\beta}\left(\frac{d r}{d \tau}\right)^{2}-r^{2}\left(\frac{d \varphi}{d \tau}\right)^{2}=S$

$e^{\alpha}\left(\frac{d t}{d \tau}\right)=E, \quad r^{2}\left(\frac{d \varphi}{d \tau}\right)=L$,

where $S=0$ in the case of photons and $S=1$ for massive test particles, $\tau$ is a canonical parameter, and $E, L$ are constants of integration. This yields

$e^{\alpha+\beta}\left(\frac{d r}{d \tau}\right)^{2}=E^{2}-e^{\alpha}\left(S+\frac{L^{2}}{r^{2}}\right)$, 

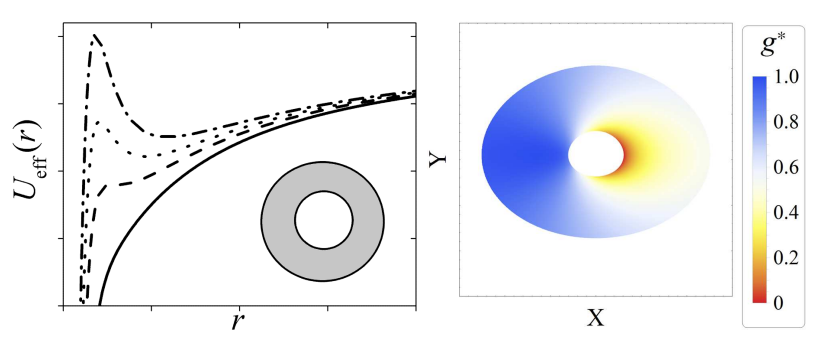

Fig. 1. Left panel: examples of $U_{\text {eff }}$ in the case of Schwarzshild-like SCO distributions shown in the right lower corner of the panel. SCOs fill the blue region that extends to infinity; in the inner white region, the circular orbits either do not exist or are unstable. Right: image of an accretion disk formed by test particles in such SCO, $M=0.5, Q=0.2$; we have chosen the maximal outer radius of the orbits $R=12$
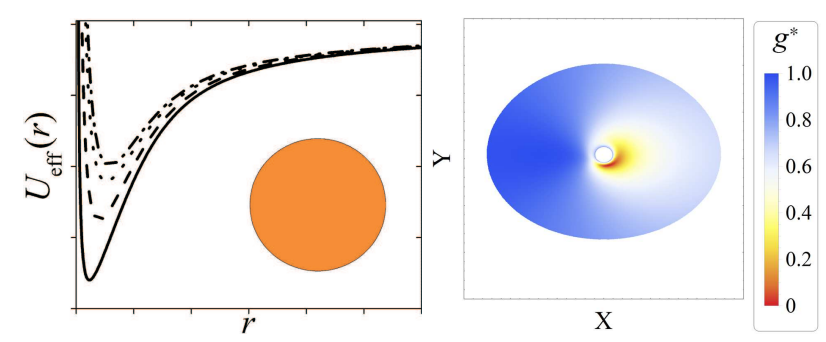

Fig. 2. Left: the same as in Fig. 1 for unbounded $U_{\text {eff }}$ with one minimum; SCOs fill all the plane including the region in the nearest vicinity of the center. Right: the image of a solid disk with the radius $R=12$ formed by test particles in these SCOs for $M=0.5, Q=0.5$
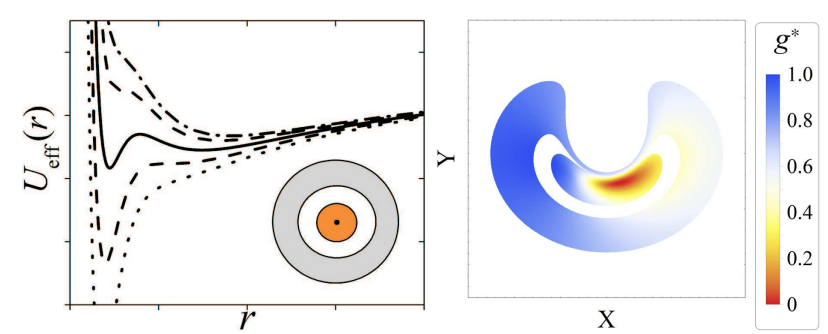

Fig. 3. Left panel: effective potentials for the geodesics in the case of a disconnected $\mathrm{SCO}$ region. Inner region (orange) includes SCO in the nearest vicinity of the center; outer SCO region (blue) extends to infinity; white ring of unstable orbits separates these regions. Right panel represents the image of the accretion disk of test particles filling this non-connected SCO distribution within the outer radius $R=12$ for $M=0.5$, $Q=1.65$. The image is deformed due to the strong deflection of rays near the singularity region. To make the ring "more round", one must take larger $R$ where
$U_{\text {eff }}(r, L)=e^{\alpha}\left(S+\frac{L^{2}}{r^{2}}\right)$,

Essentially, we have a problem of a one-dimensional particle motion in the effective potential $U_{\text {eff }}$.

Key points are related to the emergence and localization of extrema of the effective potential. In the case of the motion of test particles with nonzero masses $(S=1)$ described by the time-like trajectories, minima of $U_{\text {eff }}$ for different $L$ correspond to radii of the stable circular orbits (SCOs). We use the method of our works $[17,18]$ to study bifurcations associated with the appearance and disappearance of minima. This enables us to determine possible regions of SCOs for various solutions of the Einstein SF equations. Typical examples of $U_{\text {eff }}$, which are shown in Figs. 1-3, involve three types of possible distributions of SCOs depending on various choices of the parameters $M, Q$.

The first type (Fig. 1, left panel) corresponds to the case where $\exp [\alpha(r)] / r^{2}$ is bounded, and there is a maximum $U_{\max }=U_{\text {eff }}\left(r_{\max }\right)$ for any $L$. In this case, possible trajectories involve finite and infinite motions, including fall to the center. There is a region where either there are no circular orbits at all, or they are unstable. The regions of unstable and stable orbits for all $L$ do not overlap (the Schwarzschild-like SCO distributions).

In particular, if there exists a minimum $U_{0}=$ $=U_{\text {eff }}\left(r_{\min }\right)<1$ (for fixed $L$ ), then there is $E^{2}$ : $U_{\text {eff }}\left(r_{\text {min }}\right)<E^{2}=U_{\text {eff }}\left(r_{1}\right)=U_{\text {eff }}\left(r_{2}\right)<1, r_{1}<r_{2}$. The corresponding trajectory will oscillate around the stable circular orbit $r \equiv r_{\min }$ between radii $r_{1}$ and $r_{2}$, the angle $\varphi(\tau)$ increasing monotonically. Note that there is a minimum value of $r_{\max }<r_{\min }$ for all $L$. The trajectories with $E^{2}>U_{\max }$ describe the particle capture by the singular center. The trajectories with $1 \leq E^{2}<U_{\max }$ describe an infinite motion $(r \rightarrow \infty)$ for $\tau \rightarrow \pm \infty$.

For some choice of $M, Q$ the effective potential is unbounded for all $L$. The left panel in Fig. 2 shows examples with one potential minimum, which can have arbitrarily small $r_{\min }$. Figure 3 shows examples, when there can be two minima of $U_{\text {eff }}$ for some $L$. This represents the set of configurations (defined by $M, Q)$, when the inner and outer SCO regions are separated by a ring of unstable circular orbits.

Figure 4 shows the areas of parameters in the $M-Q$ plane with different types of the SCO dis-

ISSN 2071-0194. Ukr. J. Phys. 2019. Vol. 64, No. 11 
tributions. Here, any point of the $M-Q$ plane corresponds to a configuration representing a solution of the Einstein-SF equations satisfying the conditions at infinity.

\section{Photon Trajectories and Accretion Disk Images}

Here, we consider the simplest model of an accretion disk orbiting the configuration, which is represented by the plane SCO distribution; i.e., we assume that the "accretion disk" is formed by test particles moving along SCO in the equatorial plane within some radius $R$.

In order to build the disk images, we use the backward ray-tracing. Instead of tracking the photons emitted from the disk, we follow the photons from the observer emitted parallel to the line of sight with different impact parameters and see, where they intersect the disk.

In the case of photon trajectories $(S=0)$, the criterion for existence/non-existence of a maximum for $U_{\text {eff }}$ is the same as in the case $S=1$. If a maximum of $U_{\text {eff }}$ for photons exists, this corresponds to the Schwarzschild-like SCO distribution for massive test particles. In this case, the capture of photons incident from infinity by the singularity at the center is possible. If the singularity does not radiate anything, this means that the observer will see an image with the dark spot at the center like the case of Schwarzschild $\mathrm{BH}$. This case is depicted in Fig. 1 (right panel). Here and below, the strip at the right indicates different values of the frequency shift parameter $g^{*}=$ $=\left(\nu_{\mathrm{obs}}-\nu_{\mathrm{obs}, \min }\right) /\left(\nu_{\mathrm{obs}, \max }-\nu_{\mathrm{obs}, \min }\right)$ varying from 0 to 1 , and the inclination is $i=\pi / 4$. The white places show the images of regions, where SCOs do not exist (e.g., the white circles at the centers of Figs. 1 and 2 mean in fact that the observer will see the dark spots).

If $U_{\text {eff }}(r) \rightarrow \infty$ as $r \rightarrow 0$, this corresponds either to the distributions with stable circular orbits only (Fig. 2, left), or to the disconnected SCO distributions (Fig. 3, left). In this case, all the photons that are incident from infinity are reflected back from the potential with certain deflection angle. In the case of a small impact parameter, this angle can be large enough so that the photon does not cross the disk. This means that the light from inner orbits of the accretion disk near the center may not reach a dis-
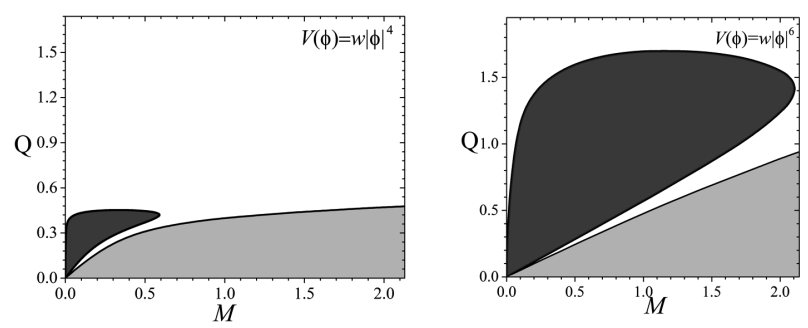

Fig. 4. Parameter areas for configurations with different SCO distributions for monomial potentials with $n=2$ and $n=3$ $(w=1)$. Light grey areas: configurations with Schwarzschildlike SCO distributions (see the left panel in Fig. 1); white areas: disconnected SCO distributions (see Fig. 3); dark grey areas: distributions with only stable circular orbits (the left panel in Fig. 2)

tant observer, depending on the mutual orientation of the line of sight and the accretion disk axis. The observer will see a dark spot at the center in this case as well, in spite of the existence of SCO close near the center (see the right panels in Figs. 2, 3).

\section{Alternative Potential}

As an alternative to the above models with monomial potentials that exclude the configurations with $\mathrm{BH}$ at the center, we consider a "toy" analytic example with a potential [17] that allows to overcome the limitations of the no-scalar-hair theorems [9,10]. Assuming the metric in the quasiglobal coordinates

$d s^{2}=A(x) d t^{2}-\frac{d x^{2}}{A(x)}-r^{2}(x) d O^{2}$,

the SF potential can be written as

$V(x)=\frac{1}{r^{2}}-\frac{A}{r^{2}}\left(3\left(r^{\prime}\right)^{2}+r r^{\prime \prime}\right)+2 \frac{x-3 M}{r^{3}} \frac{d r}{d x}$,

where

$r(x)=x\left[1-\left(\frac{x_{0}}{x}\right)^{P}\right], \quad x>0$,

$P>2$ and $x_{0}>0$ are constant parameters, and

$A(x)=(1-y)^{2}\left\{F[4, a, b, y]-\frac{2 M}{x} F[4, c, d, y]\right\}$,

$a=2 / P, b=1+a, c=3 / P, d=1+c ; y=\left(x_{0} / x\right)^{P}$;

$\phi(x)= \pm \sqrt{\frac{8(P-1)}{P}} \arcsin \left[\left(\frac{x_{0}}{x}\right)^{P / 2}\right]$. 


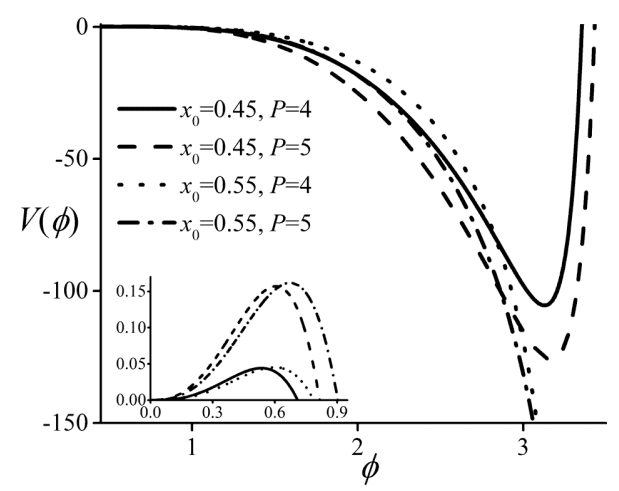

Fig. 5. Typical examples of the scalar field potentials (12) in the cases of $\mathrm{BH}$ and NS. In the left lower corner, we show the behavior of near the central minimum
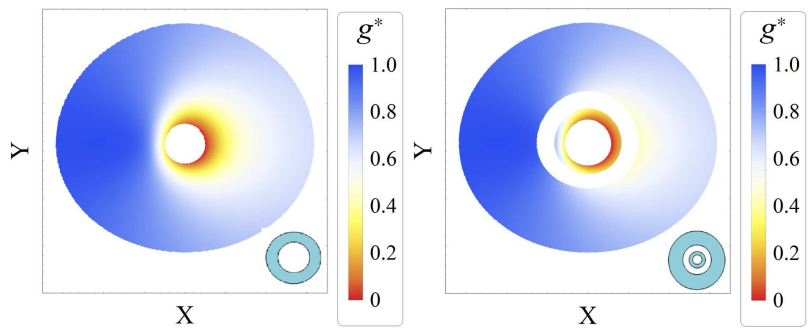

Fig. 6. Case of potential 12, the BH configurations: images of an accretion disk formed by test particles in SCO within the outer radius $R=3, M=1 / 6$. Left: the Schwarzschild-like distributions, $x_{0}=0.45, P=4$; right: non-connected SCO distribution, $x_{0}=0.45, P=5$. In both cases and in the figures below, the inclination angle is $i=\frac{\pi}{4}$
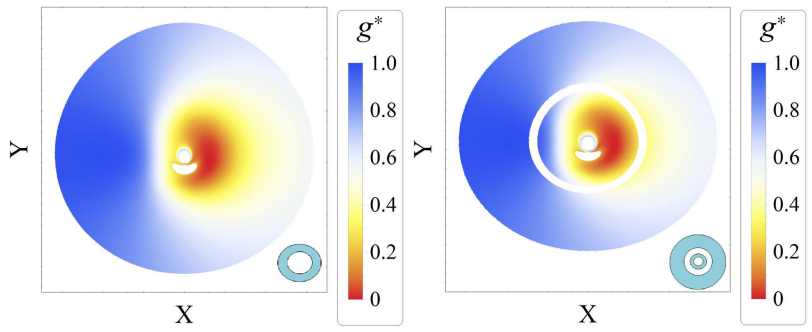

Fig. 7. Case of potential 12, the NS configurations: images of an accretion disk formed by test particles in SCO within the outer radius $R=3, M=1 / 6$. As distinct from the $\mathrm{BH}$ case, there are additional features near the image centers. Left panel shows the case where SCOs that form the accretion disk begin with non-zero radii, $x_{0}=0.6, P=4$; right: the disk is formed by a non-connected SCO distribution, $x_{0}=0.6, P=5$

Formulas (13)-(15) represent a family of static spherically symmetric asymptotically flat configurations that correspond to potentials (12). These configurations are completely defined by parameters
$M, P, x_{0}$. The center of each configuration in the curvature coordinates $(r=0)$ corresponds to $x=x_{0}$.

It was shown [17] that, for $x_{0} \geq 3 M$, the solutions yield the naked singularity (NS) at the center $x=x_{0}$; and, for $0<x_{0}<3 M$, we have $\mathrm{BH}$ with a horizon at some $x=x_{h}>x_{0}$. In the latter case, the SF potential (12) has the form like a "Mexican hat" with an infinite (unbounded from above) edges; in the case of $x_{0} \geq 3 M$, the SF potential is unbounded from below (Fig. 5). We have two possible types of the SCO distributions that can be found in [17]. The first one is similar to that depicted in Fig. 1. The second differs from Fig. 3 by the existence of an additional central region analogous to that of the Schwarzschild-like case where circular orbits are either unstable or do not exist (see the ring-like structures in the right lower corners of the panels). Examples of the corresponding accretion disk images are shown in Figs. 6 and 7.

\section{Conclusions}

We have investigated static spherically symmetric solutions of the joint system of Einstein SF equations assuming Lagrangian (1) with two types of the SF potentials. The solutions with the SF potential (2) contain the naked singularity at the center; on the other hand, the analytic solution of Section 6 admits, for some parameters, the black hole configuration (though potential (12) is rather exotic). We built the SCO distributions and analyzed the images of accretion discs (represented by these distributions in one plane) observed from infinity.

Obviously, the studies of real accretion disks around astrophysical objects should consider complex (and poorly known) physical conditions such as the gas pressure, magnetic fields, disk inhomogeneities, turbulence, etc. However, their detailed investigation in the context of our paper would be premature. On the other hand, the consideration of stable or unstable circular orbits, which is only the first step to this problem, reveals the main features that are important in connection with the observed EHT image.

Possible types of the SCO distributions and their images are shown in Figs. 1, 2, 3, 6, and 7. The principal question is how to distinguish these images from those of accretion disks around standard black holes. In this paper, we do not consider the 
full-scale simulations in order to reproduce the observed EHT image [1]; however, on the qualitative level, we see that there is a lot of possibilities to fix parameters of the $\mathrm{BH}$ mimickers so as to reproduce the typical picture containing a luminous ring with a dark spot at the center - similar to the case of the Schwarzschild BH. It is interesting to note that such a picture can occur even in the case of a solid accretion disk (see Fig. 2), but not for all disk sizes and orientations. Further developments must involve the geometry and distribution of the surface brightness over the accretion disk, which may be appropriate under better resolution. On the other hand, for some model parameters, there can be significant differences of images such as in the presence of the separation of SCO (Fig. 3, 6, 7, right panels); this can be used to rule out some parameter areas of the models.

This work has been supported by the scientific program "Astronomy and space physics" (project 19БФ023-01) of Taras Shevchenko National University of Kyiv.

1. Event Horizon Telescope Collaboration. First M87 event horizon telescope results. I. The shadow of the supermassive Black Hole. ApJ Lett. 875, id. L1 (2019).

2. Event Horizon Telescope Collaboration. First M87 event horizon telescope results. V. Physical origin of the asymmetric ring. ApJ Lett. 875, id. L5 (2019).

3. Y. Mizuno et al. The current ability to test theories of gravity with Black Hole shadows. Nat. Astron. 2, 585 (2018).

4. C.M. Will. The confrontation between general relativity and experiment. Liv. Rev. Relativ. 17, 4 (2014) [arXiv:1403.7377].

5. E. Berti, E. Barausse, V. Cardoso et al. Testing general relativity with present and future astrophysical observations. Class. Quant. Grav. 32, 243001 (2015) [arXiv: 1501.07274].

6. A. Linde. Inflationary cosmology. Lect. Notes Phys. 738, 1 (2008) [arXiv:0705.0164].

7. B. Novosyadlyi, V. Pelykh, Yu. Shtanov, A. Zhuk. Dark Energy and Dark Matter of the Universe (In three volumes). Vol. 1. Dark Matter: Observational Evidence and Theoretical Models. Ed. V. Shulga (Akademperiodyka, 2013) [arXiv:1502.04177].

8. K. Bamba, S. Capozziello, S. Nojiri, S.D. Odintsov. Dark energy cosmology: The equivalent description via different theoretical models and cosmography tests. Ap. Sp. Sci. 342, 155 (2012) [arXiv:1205.3421].

ISSN 2071-0194. Ukr. J. Phys. 2019. Vol. 64, No. 11
9. J.D. Bekenstein. Transcendence of the law of baryonnumber conservation in black-hole physics. Phys. Rev. Lett. 28, 452 (1972).

10. J.D. Bekenstein. Nonexistence of baryon number for black holes. II. Phys. Rev. D 5, 1239, 2403 (1972).

11. I.Z. Fisher. Scalar mesostatic field with regard for gravitational effects. Zh. Exp. Theor. Phys. 18, 636-640 (1948) [arXiv:gr-qc/9911008].

12. A.I. Janis, E.T. Newman, J. Winicour. Reality of the Schwarzschild singularity. J. Phys. Rev. Lett. 20, 878 (1968).

13. M. Wyman. Static spherically symmetric scalar fields in general relativity. Phys. Rev. D 24, 839 (1981).

14. K.S. Virbhadra. Janis-Newman-Winicour and Wyman solutions are the same. Int. J. Mod. Phys. A 12, 4831 (1997).

15. R.A. Asanov. Point source of massive scalar field in gravitational theory. Teor. Matem. Fiz. 20, 1, (1974).

16. O.S. Stashko, V.I. Zhdanov. Disconnected regions of stable circular orbits in presence of massive scalar field. Odessa Astron. Publ. 30, (2017).

17. O.S. Stashko, V.I. Zhdanov. Spherically symmetric configurations of General Relativity in presence of scalar fields: separation of circular orbits. Gen. Rel. Grav. 50, id. 105 (2018).

18. O.S. Stashko, V.I. Zhdanov. Spherically symmetric configurations in general relativity in the presence of a linear massive scalar field: Separation of a distribution of test body circular orbits. Ukr. J. Phys. 64, No. 3, 189 (2019).

19. D. Solovyev, A. Tsirulev. General properties and exact models of static self-gravitating scalar field configurations. Classic. Quant. Grav. 29, id. 055013 (2012).

20. Z. Stuchlik, J. Schee. Appearance of Keplerian discs orbiting Kerr superspinars. Classic. Quant. Grav. 27, id. 215017 (2010) [arXiv:1101.3569].

21. A.N. Chowdhury, M. Patil, D. Malafarina, P.S. Joshi. Circular geodesics and accretion disks in Janis-NewmanWinicour and Gamma metric. Phys. Rev. D 85, id. 104031 (2012) [arXiv:1112.2522].

22. R.S.S. Vieira, J. Schee, W. Kluźniak, Z. Stuchlík. Circular geodesics of naked singularities in the Kehagias-Sfetsos metric of Horava's gravity. Phys. Rev. D 99 id. 024035 (2014) [arXiv:1311.5820].

23. K. Boshkayev, E. Gasperin, A.C. Gutierrez-Pineres, H. Quevedo, S. Toktarbay. Motion of test particles in the field of a naked singularity. Phys. Rev. D 93, id. 024024 (2016) [arXiv:1509.03827].

24. V.V. Nikonov, Ju.V. Tchemarina, A.N. Tsirulev. A twoparameter family of exact asymptotically flat solutions to the Einstein-scalar field equations. Classic. Quant. Grav. 25, id. 138001 (2008).

Received 29.08.19 
О.С. Сташко, В.І. Жданов

ІМІТАТОРИ ЧОРНОЇ ДІРИ

В АСТРОФІЗИЧНИХ КОНФІГУРАЦІЯХ

ЗІ СКАЛЯРНИМИ ПОЛЯМИ

$\mathrm{P}$ е $з$ ю м е

Досліджено статичні сферично-симетричні конфігурації в ЗТВ у присутності мінімально-зв'язаного скалярного поля (СП). Для мономного потенціалу самодії СП, розв'язки системи рівнянь Айнштайна-СП отримані чисельно, після цього отримано розподіл стійких колових орбіт та зображення "акреційного диску" з точки зору віддаленого спостерігача. Розглянуто аналогічну задачу на прикладі аналітичного розв'язку зі спеціально підібраним потенціалом СП, що допускає існування чорної діри. Показано, що у багатьох випадках зображення практично такі ж, як і у випадку чорної діри Шварцшильда з яскравим кільцем і темною плямою в центрі. 3 іншого боку, певний вибір параметрів приводить до специфічних особливостей у зображеннях, які можуть допомогти виключити деякі екзотичні моделі. 